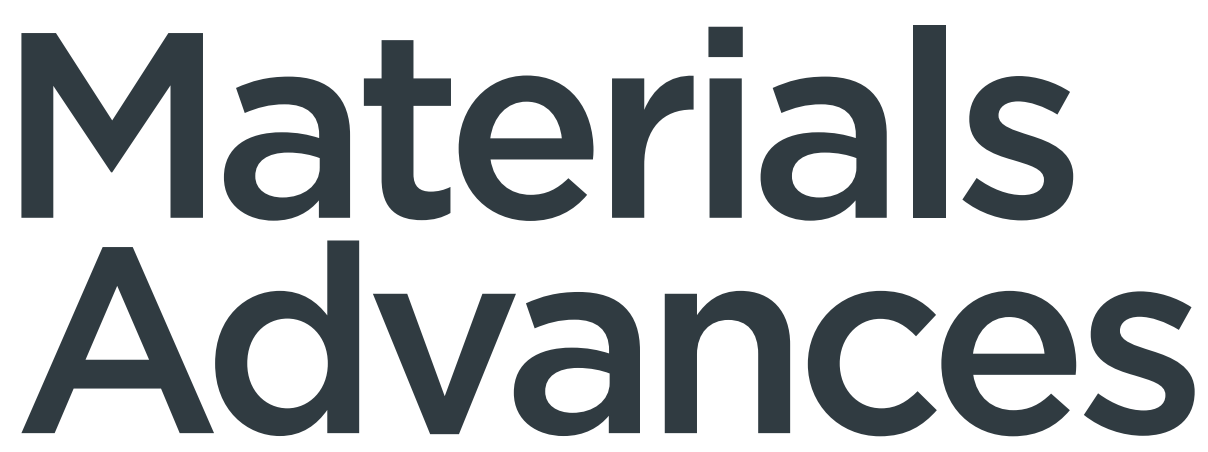

Number 17

7 September 2021

Pages 5503-5792

rsc.li/materials-advances

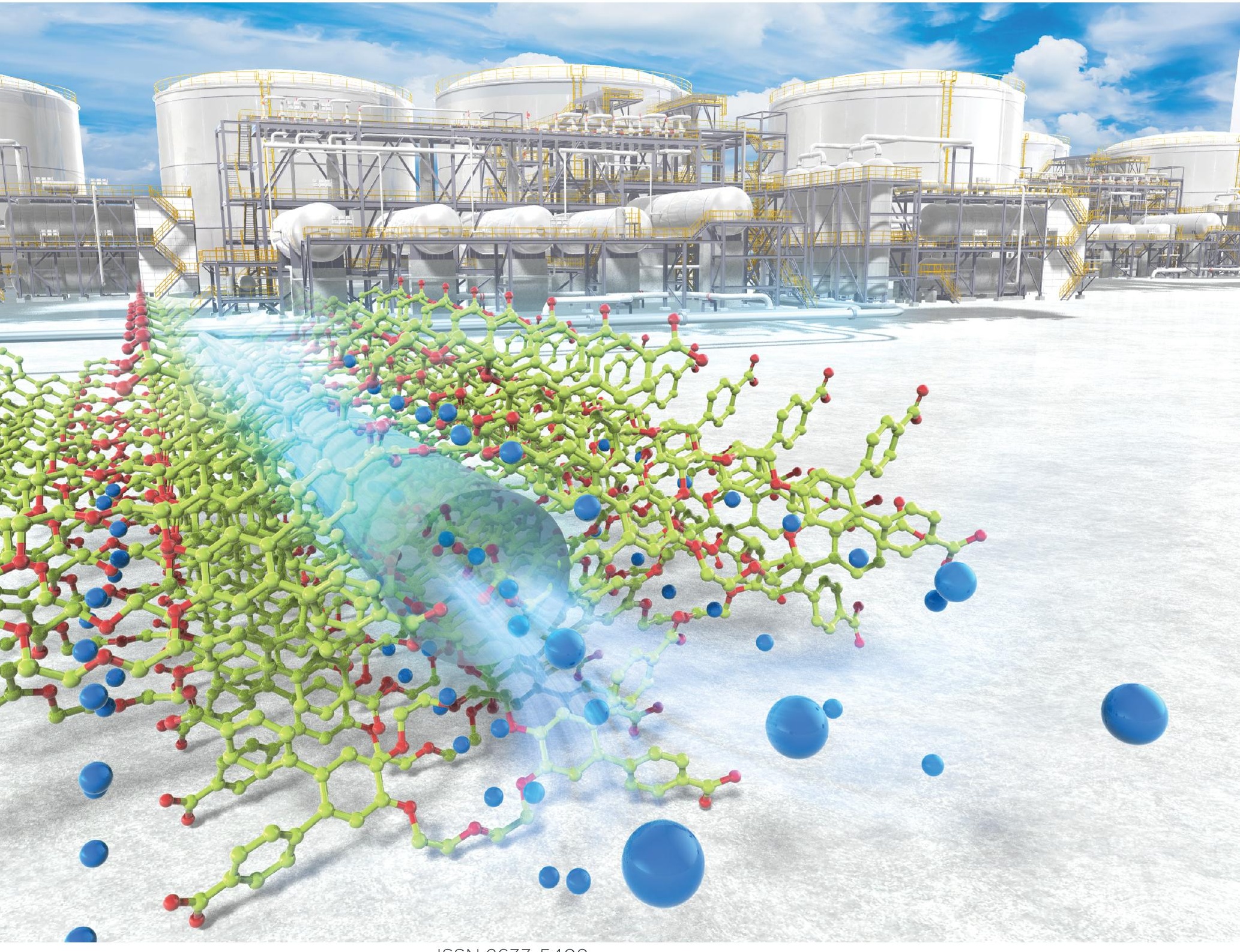

ISSN 2633-5409 
Check for updates

Cite this: Mater. Adv., 2021, 2, 5639

Received 6th May 2021,

Accepted 29th June 2021

DOI: $10.1039 / \mathrm{d} 1 \mathrm{ma} 00411 \mathrm{e}$

rsc.li/materials-advances

\section{A proton conductive hydrogen-bonded framework incorporating 18-crown-6-ether and dicarboxy-o-terphenyl moieties $\dagger$}

\author{
Xin Chen, ${ }^{a}$ Kiyonori Takahashi, (D) *ab Kenta Kokado, (D) ab Takayoshi Nakamura*ab \\ and Ichiro Hisaki iD *abc
}

To date, proton-conducting organic crystalline materials based on crown ethers have rarely been investigated. In this work, we reveal that flexible organic 18-crown-6 derivatives with one or two 4 , $4^{\prime}$-dicarboxy-O-terphenyl (CT) groups formed four kinds of crystalline frameworks: 1 CT-18C6-I $\left(P 2_{1} / n\right)$, 2CT-18C6-I $(P \overline{1}), 2$ 2CT-18C6-II $(P \overline{1})$ and 2CT-18C6-III $\left(C m c 2_{1}\right)$. Single crystal X-ray diffraction analysis clearly suggested that the water molecules were involved a hydrogen-bonded network for two frameworks. In particular, a unique one-dimensional (1D) water pathway had formed in 2CT-18C6-III and the activation energy was evaluated by Arrhenius plots to be $0.14 \mathrm{eV}$, indicating that the proton jumps from $\mathrm{H}_{3} \mathrm{O}^{+}$to the neighboring $\mathrm{H}_{2} \mathrm{O}$ in the hydrogen-bonded network of 2CT-18C6-III.

\section{Introduction}

Under the concept of green sustainable development, new clean fuel sources are absolutely necessary. Proton exchange membrane fuel cells (PEMFCs) are one of the most optimal technologies as an alternative to common fossil fuels. ${ }^{1}$ Proton exchange membranes (PEMs) with an ionic pathway for protons are critical for PEMFCs. Presently, organic conductive polymers, such as Nafion, sulfonated and phosphonic-based polyaromatics, and polybenzimidazoles, are employed as alternative PEM materials. ${ }^{2-7}$ They exhibit the following features required for PEMs: (a) the presence of proton-conducting functionalities, ${ }^{8-10}$ (b) poor electronic conductivities, and (c) good chemical and thermal stabilities. However, even the Nafion, achieving the outstanding proton conductivity of $10^{-1} \mathrm{~S} \mathrm{~cm}^{-1}$ at room temperature with $100 \%$ relative humidity $(\mathrm{RH})$, still involves unavoidable defects. Thus, exploration of optimizing electrolyte materials has attracted much attention in this field. Highly crystalline materials with a periodic framework and infinite channels such as porous coordination polymers (PCPs) and metal-organic

\footnotetext{
${ }^{a}$ Graduate School of Environmental Science, Hokkaido University, Sapporo, Hokkaido 060-0810, Japan

${ }^{b}$ Research Institute for Electronic Science, Hokkaido University, Sapporo, Hokkaido 001-0020, Japan.E-mail: ktakahashi@es.hokudai.ac.jp, tnaka@es.hokudai.ac.jp 'Graduate School of Engineering Science, Osaka University, 1-3 Machikaneyama, Toyonaka, Osaka 560-8531, Japan.E-mail: hisaki@chem.es.osaka-u.ac.jp $\dagger$ Electronic supplementary information (ESI) available: Synthesis of compounds, crystal data, crystal structure of 2CT-18C6-II and impedance spectroscopy of frameworks. CCDC 2057700, 2078832, 2078834 and 2078835. For ESI and crystallographic data in CIF or other electronic format see DOI: 10.1039/d1ma00411e
}

frameworks (MOFs) are, therefore, promising candidates for proton conductive materials. ${ }^{11}$

Proton conduction in PEMs is related to two mechanisms, namely, the Grotthuss and vehicle mechanisms. ${ }^{12-14}$ The vehicle mechanism indicates the diffusion of protons with the vehicles. However, the Grotthuss mechanism always occurs in a water-based degenerate system consisting of $\mathrm{H}_{3} \mathrm{O}^{+}$and $\mathrm{H}_{2} \mathrm{O}$ within an infinite network of hydrogen bonds. A proton jumps from $\mathrm{H}_{3} \mathrm{O}^{+}$to a neighbouring $\mathrm{H}_{2} \mathrm{O}$ as molecule rotations taking place, which contribute to a high proton conductivity.

As is well known, crown ether derivatives, such as dibenzo18-crown-6-ether (DB18C6) with an excellent ability to bind alkali metal cations and water molecules, ${ }^{15,16}$ have drawn much attention as an ion conductive material. ${ }^{17}$ Theoretical calculation indicates that DB18C6 with $\mathrm{H}_{3} \mathrm{O}^{+}$ions located in the cavities of the crown ether interact with water molecules. ${ }^{18,19}$ Therefore, crystalline frameworks composed of crown ethers are expected to show conductivity with the Grotthuss mechanism. In this connection, we were interested in hydrogenbonded organic frameworks (HOFs), ${ }^{20-25}$ in which molecules are connected through non-covalent intermolecular interactions such as hydrogen bonds, because of their high crystallinity and high affinity toward protons. To date, some HOFs have been reported to show proton conductivity. ${ }^{26-32}$ However, proton conductive HOFs based on crown ethers are hitherto unknown.

Herein, we newly designed 18-crown-6 derivatives with one and two 4,4'-dicarboxy-o-terphenyl (CT) groups, 1CT-18C6 and 2CT-18C6, respectively, as building block molecules to develop proton conductive HOFs (Fig. 1). A working hypothesis is as 
(a)

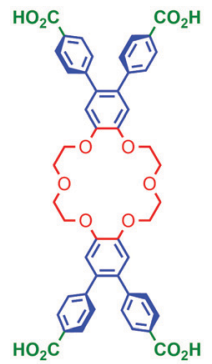

(b)

2CT-18C6

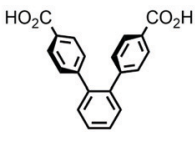

CT
H-bonding group : carboxy group

- network formation

-water molecule capture

spacer : o-terphenyl - space for conductive pathway - framework sckeleton

ionopher : crown ether -ions pathway
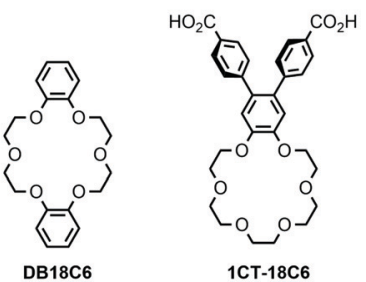

Fig. 1 (a) The molecular structure of 2CT-18C6 and expected roles of the parts. (b) Related molecules 1CT-18C6, dibenzo-18-crown-6 (DB18C6), and 4,4'-dicarboxy-o-terphenyl (CT).

follows: (1) the 18-crown-6 and carboxy groups can capture water molecules via hydrogen bonds; (2) the carboxy group also can form supramolecular synthons to organize the molecules into crystalline supramolecular frameworks; ${ }^{33}$ and (3) the rigid $o$-terphenyl moiety can form frameworks ${ }^{24,34-36}$ and provide space for proton conduction. To date, 2CT-18C6 has been applied as an organic ligand of MOFs, ${ }^{37}$ and proton conductive molecular crystalline materials based on 2CT-18C6 are hitherto-unknown.

2CT-18C6 was revealed to form three kinds of crystalline frameworks (2CT-18C6-I, -II, and -III). In particular, 2CT-18C6-III has a unique one-dimensional (1D) water pathway and showed a proton conductivity of $6.75 \times 10^{-8} \mathrm{~S} \mathrm{~cm}^{-1}$ at $85 \% \mathrm{RH}$. Notably, the activation energy $\left(E_{\mathrm{a}}\right)$ was evaluated by Arrhenius plots to be $0.14 \mathrm{eV}$, suggesting that the proton jumps from $\mathrm{H}_{3} \mathrm{O}^{+}$to the neighbouring $\mathrm{H}_{2} \mathrm{O}$ in the hydrogen-bonding networks of 2CT18C6-III. Thermogravimetric (TG) analysis also indicated that 2CT-18C6-III is stable up to $588 \mathrm{~K}$. The present system is the first example of the tailoring of structural features of crown ethers with a $1 \mathrm{D}$ water pathway.

In this paper, we present the synthesis of crown ether derivatives, construction of crystalline frameworks, and evaluation of their proton conductivity. Moreover, the relationships between the structures of water pathways and proton conductivity are discussed.

\section{Results and discussion}

\section{Synthesis and crystallization}

Synthesis of 1CT-18C6 and 2CT-18C6 is shown in Scheme 1a. 4, 5-Bis(methoxycarbonylphenyl)benzo-18-crown-6 (1) and 4,4' $, 5,5^{\prime}$-tetrakis(methoxycarbonylphenyl)dibenzo-18-crown-6 (2) were synthesized through a Suzuki-Miyaura cross-coupling reaction of (4-(methoxycarbonyl)phenyl)boronic acid and the corresponding brominated crown ether derivatives. Hydrolysis of 1 and 2 gave 1CT-18C6 and (a)
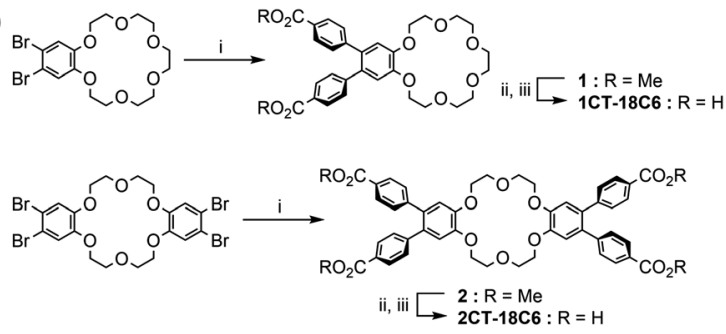

(b)

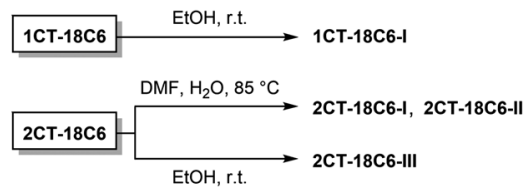

Scheme 1 (a) Synthesis and (b) crystallization of 1CT-18C6 and 2CT18C6. Reaction conditions: (i) 4-(methoxycarbonyl)phenylboronic acid, $\mathrm{Pd}\left(\mathrm{PPh}_{3}\right)_{4}, \mathrm{~K}_{2} \mathrm{CO}_{3}, \mathrm{THF}$, water, reflux; (ii) $\mathrm{NaOH}_{\text {aq }}$. THF, (iii) $\mathrm{HCl}$.

2CT-18C6, respectively. Conditions to prepare the frameworks are shown in Scheme 1b. 1CT-18C6 dissolved in $\mathrm{EtOH}$ at room temperature for 1 day afforded colourless block crystals (1CT-18C6-I). 2CT-18C6 forms three forms 2CT-18C6-I, -II, and -III. $\$$ Slow evaporation of a mixed solution of $N, N$-dimethylformamide (DMF) and water at $85{ }^{\circ} \mathrm{C}$ for several days successfully obtained two kinds of block-shaped $P \overline{1}$ crystals (2CT-18C6-I and 2CT-18C6-II) concomitantly. 2CT-18C6 dissolved in EtOH at room temperature afforded a colourless block $\mathrm{Cmc2}_{1}$ crystal (2CT-18C6-III).

\section{Crystallography}

In a 2CT-18C6-I crystal, a sodium cation is captured at the centre of the crown ether with a $\mathrm{Na}^{+} \ldots \mathrm{O}$ distance ranging from 2.57 to $2.79 \AA$ A . Two DMF molecules are then coordinated to $\mathrm{Na}^{+}$ from axial directions. Because of the cation binding, the $18 \mathrm{C} 6$ moiety has a boat-shaped conformation with a bending angle of $76.4^{\circ}$ (Fig. 2a), where the bending angle is defined as a dihedral angle between the mean planes of the two benzene rings of the dibenzo-18-crown-6-ether moiety. Three of the four carboxy groups form intermolecular hydrogen bonds and one binds to a DMF molecule, resulting in the formation of a ribbon like

$\ddagger$ Crystal data for 1CT-18C6-I, $\mathrm{C}_{30} \mathrm{H}_{36} \mathrm{O}_{13} ; F_{\mathrm{w}}=604.59$; monoclinic, $P 2_{1} / n$ (\#14), $Z=4, a=13.6633(2) \AA, \quad b=8.2601(1) \AA, c=26.8789(6) \AA, \beta=90.208(2)^{\circ}, V=3033.54(9)$ $\AA^{3}, T=223 \mathrm{~K}, D=1.324 \mathrm{~g} \mathrm{~cm}^{-3}, 12904$ collected, 5424 unique $\left(R_{\text {int }}=0.031\right)$ reflections, and the final $R_{1}$ and $\mathrm{w} R_{2}$ values are $0.059[I>2.0 \sigma(I)]$ and 0.184 (all data), respectively. CCDC 2057700. Crystal data for 2CT-18C6-I, $\mathrm{C}_{58.5} \mathrm{H}_{64.5} \mathrm{~N}_{3.5} \mathrm{Na}$ $\mathrm{O}_{17.5} ; F_{\mathrm{W}}=1119.62$; triclinic, $P \overline{1}(\# 2), Z=2, a=11.3113(2) \AA$ А $, b=13.4267(2) \AA, c=$ 21.7047(4) ̊, $\alpha=104.103(2)^{\circ}, \beta=95.267(1)^{\circ}, \gamma=111.048(2)^{\circ}, V=2924.57$ (10) $\AA^{3}$, $T=293 \mathrm{~K}, D=1.271 \mathrm{~g} \mathrm{~cm} \mathrm{~cm}^{-3}, 31008$ collected, 11017 unique $\left(R_{\mathrm{int}}=0.026\right)$ reflections, and the final $R_{1}$ and $\mathrm{w} R_{2}$ values are $0.061[I>2.0 \sigma(\mathrm{I})]$ and 0.209 (all data), respectively. CCDC 2078832. Crystal data for 2CT-18C6-II, $\mathrm{C}_{57} \mathrm{H}_{60} \mathrm{~N}_{3} \mathrm{NaO}_{17} ; F_{\mathrm{w}}$ = 1082.07; triclinic, $P \overline{1}$ (\#2), $Z=6, a=13.1094(8) \AA ̊, ~ b=25.3405(16) \AA, c=26.7457(16)$ $\AA, \alpha=105.281(5)^{\circ}, \beta=90.128(5)^{\circ}, \gamma=91.834(5)^{\circ}, V=8565.8(9) \AA^{3}, T=293 \mathrm{~K}, D=$ $1.259 \mathrm{~g} \mathrm{~cm}^{-3}, 89237$ collected, 32350 unique $\left(R_{\text {int }}=0.116\right)$ reflections, and the final $R_{1}$ and $\mathrm{w} R_{2}$ values are $0.081[I>2.0 \sigma(\mathrm{I})]$ and 0.352 (all data), respectively. CCDC 2078834. Crystal data for 2CT-18C6-III, $\mathrm{C}_{48} \mathrm{H}_{42} \mathrm{O}_{17} ; F_{\mathrm{w}}=890.81$; orthorhombic, $C m c 2_{1}$ (\#36), $Z=4, a=27.4687(10) \AA, b=20.3236(8) \AA, c=8.0251(6) \AA$, $V=4480.1(4) \AA^{3}, T=298 \mathrm{~K}, D=1.321 \mathrm{~g} \mathrm{~cm}^{-3}, 29283$ collected, 4181 unique $\left(R_{\mathrm{int}}=0.128\right)$ reflections, and the final $R_{1}$ and $\mathrm{w} R_{2}$ values are $0.094[I>2.0 \sigma(I)]$ and 0.288 (all data), respectively. CCDC 2078835 . 

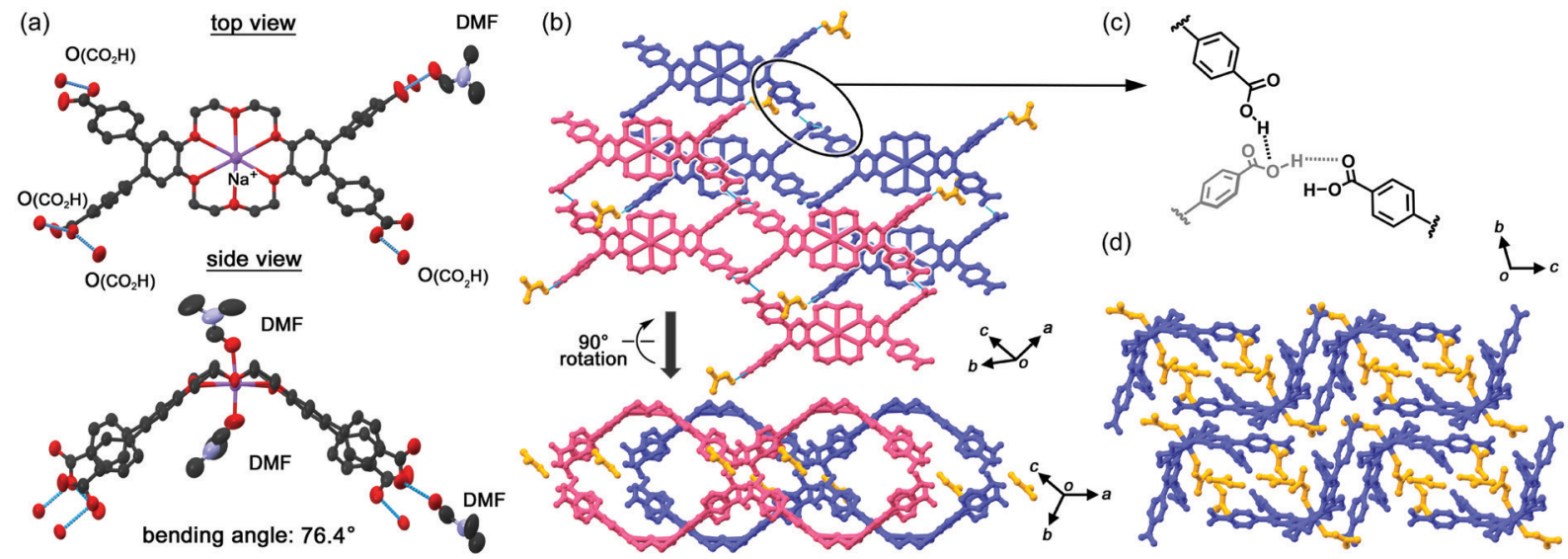

Fig. 2 Crystal structure of 2CT-18C6-I. (a) Anisotropic ellipsoid plot with 50\% probability. (b) One-dimensional ribbon-like motifs formed through intermolecular hydrogen bonds. (c) Branched hydrogen-bonded network formed by three carboxy groups. (d) Packing diagram, where the crown ether moiety and DMF molecules are coloured by purple and orange, respectively.

motif (Fig. 2b). It is noteworthy that the carboxy groups form no self-complementary dimer, which is often observed in hydrogen-bonded frameworks composed of highly-symmetric rigid $\pi$-conjugated molecules, ${ }^{22-25}$ but branched networks as shown in Fig. 2c. This result clearly indicates that the flexibility and lower symmetry of 2CT-18C6-I crucially affect the route to the formation of a hydrogen-bonded network. Packing of the boat shaped molecules provides small inclusion spaces, in which DMF molecules are included. In this framework, however, there is no $1 \mathrm{D}$ water alignment suitable for proton conduction. 2CT-18C6-II consists of three crystallographically independent molecules as well as DMF molecules. 2CT-18C6-II has almost the same structure as 2CT-18C6-I (Fig. S1, ESI $\dagger$ ).

In the 2CT-18C6-III crystal, a Cs-symmetric 2CT-18C6 molecule has quite a shallow boat conformation with a bending angle of $25.2^{\circ}$ (Fig. 3a), which is significantly different from 2CT-18C6-I. The observed differences in the conformation are probably caused by the existence of strongly bound cationic species in the macrocycle. The carboxy groups form hydrogen bonds with only water molecules to give the crystal, which contains no solvent but water molecules with a host/guest ratio of 1:3 stoichiometry (Fig. 3b). One water molecule forms hydrogen bonds with the crown ether's oxygen and the carboxy groups with a distance of 2.86 and $2.69 \AA$, respectively, and is isolated from neighbouring water molecules (yellow spheres). The other four molecules (red spheres), on the other hand, form hydrogen bonds with the carboxy groups $(\mathrm{O} \cdots \mathrm{O}$ distances: $2.72,2.89$, and $2.94 \AA$ ) to form a 1D zig-zag alignment along the $c$ axis (Fig. 3c). The distance between the oxygen atoms of the neighbouring water molecules is 2.97 and $3.74 \AA$ (Fig. 3d).

1CT-18C6-I also contains only water molecules with a host/ guest ratio of a $1: 3$ stoichiometry. Two water molecules are hydrogen bonded by the crown ether's oxygen atoms and the others are by the carboxy groups. In total, the water molecules are aligned one dimensionally along the $b$ axis. The distance between oxygen atoms of the neighbouring water molecules ranges from 2.77 to $3.68 \AA$. Although these distances are relatively shorter than that in the case of 2CT-18C6-III, the alignment crosses through the crown ether ring (Fig. 4).

\section{Phase purity and thermal stability}

The powder X-ray diffraction (PXRD) patterns of crystalline bulk samples of 1CT-18C6-I and 2CT-18C6-III are in good agreement
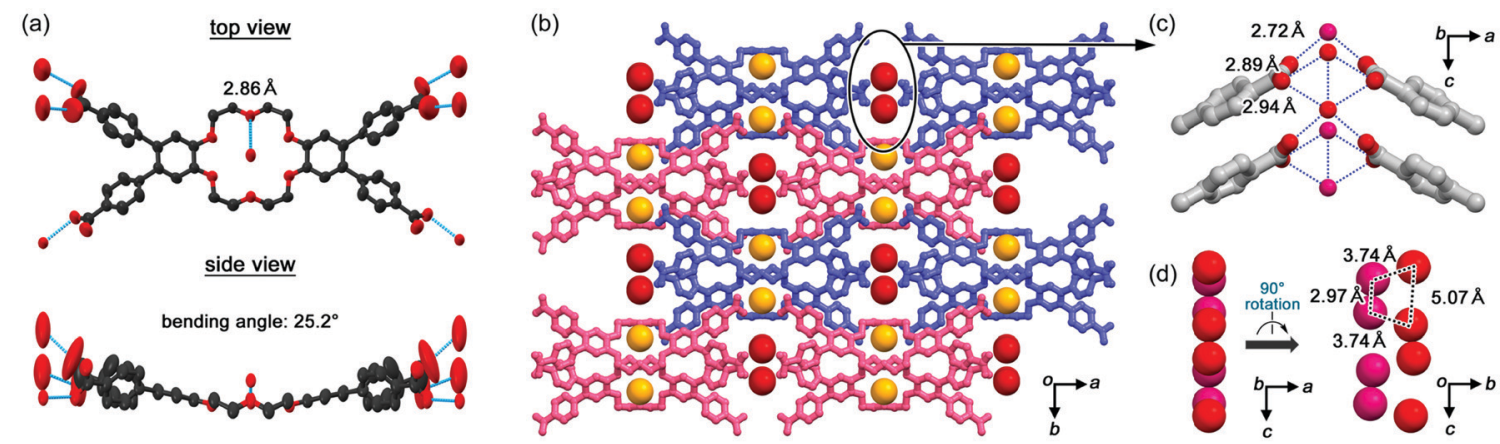

Fig. 3 Crystal structure of 2CT-18C6-III. (a) Anisotropic ellipsoid plot with 50\% probability. (b) Packing diagram, where two types of water molecules are coloured with red and orange. (c) Hydrogen bonds among carboxy groups and water molecules. (d) Alignment of water in the channel with intermolecular distances. 

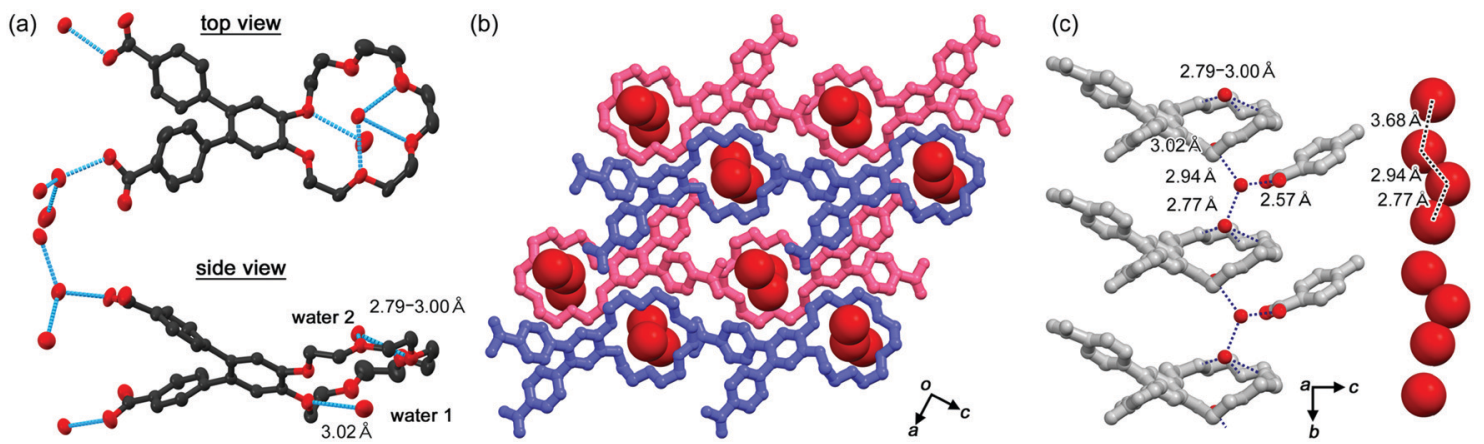

Fig. 4 Crystal structure of 1CT-18C6-I. (a) Anisotropic ellipsoid plot with 50\% probability. (b) Packing diagram, where water molecules are shown by the space-fill model. (c) Hydrogen bonds among the crown ether, carboxy groups, and water molecules. (d) Alignment of water in the channel with intermolecular distances.

with the ones simulated from their single-crystal data respectively (Fig. 5), indicating high phase purity and good stability. In the TG curves, 1CT-18C6-I starts losing slowly from room temperature and displays $8.9 \%$ weight loss to $373 \mathrm{~K}$, which corresponds to the loss of the water molecules (cal. 8.9\%). Then the framework is maintained until $588 \mathrm{~K}$ (Fig. 5a). Similarly, 2CT-18C6-III shows a $4.5 \%$ weight loss of water molecules from room temperature to $373 \mathrm{~K}$ (cal. 4.5\%) and the framework also collapses after $588 \mathrm{~K}$ (Fig. $5 \mathrm{~b}$ ). On the other hand, variable temperature-PXRD plots show both 1CT-18C6-I and 2CT-18C6III with an obvious change of structure with the increase of the temperature, which result from the reorganization of the hydrogen bonds connected with the loss of the water molecules (Fig. 5c and d).

\section{Proton conductivity}

The structures of 1CT-18C6-I and 2CT-18C6-III were constructed by a hydrophilic crown ether moiety, assembly of carboxy groups, and 1D alignment of water molecules. In addition, TG and variable temperature-PXRD analyses suggested the frameworks of 1CT-18C6-I and 2CT-18C6-III were maintained after the removal of water molecules. These results indicated that there were enough sites for water molecules supplied at a higher relative humidity. These structural features could provide an orderly route for proton transport. Therefore, 1CT-18C6-I and 2CT-18C6-III are expected to show proton conductivity. Due to the limitation of the size of the samples, a compressed pellet of the samples was used for the measurement for proton conductivity. As a reference, proton conductivity under the same conditions was measured for pelletized samples of CT and DB18C6.

Temperature-dependent Nyquist plots under $85 \% \mathrm{RH}$ for four samples of 1CT-18C6-I, 2CT-18C6-III, CT, and DB18C6 are summarized in Fig. 6a and b, Fig. S3, and S4 (ESI $\dagger$ ), respectively.

The Nyquist plots (Fig. $6 \mathrm{a}$ and $\mathrm{b}$ and Fig. S3, ESI $\dagger$ ) of 1CT18C6-I, 2CT-18C6-III, and CT are the sum of a straight line and a semicircle in the low and high frequency range, respectively.

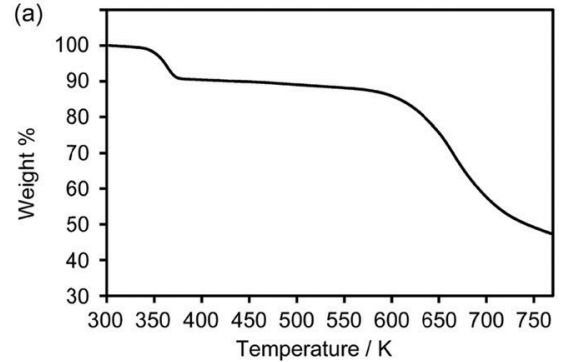

(c)

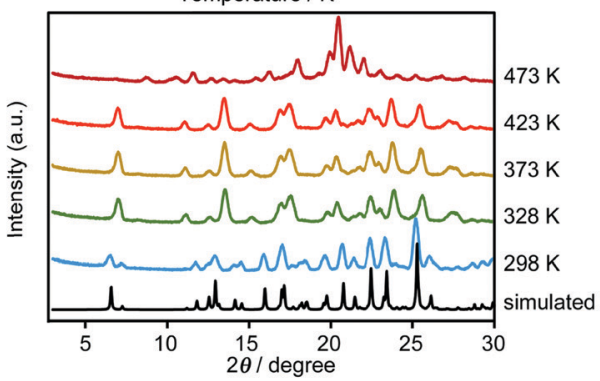

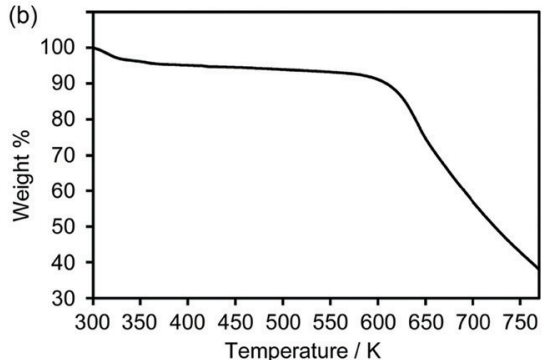

(d)

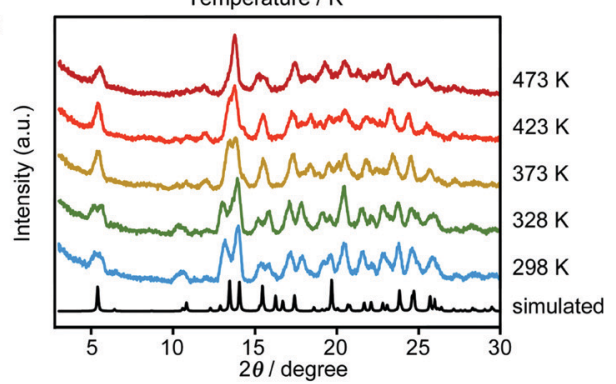

Fig. 5 TG plots of as-formed crystals of (a) 1CT-18C6-I and (b) 2CT-18C6-III. Variable temperature PXRD patterns of (c) 1CT-18C6-I and (d) 2CT-18C6-III, where simulated patterns based on SXRD analysis are shown at the bottom. 
(a)

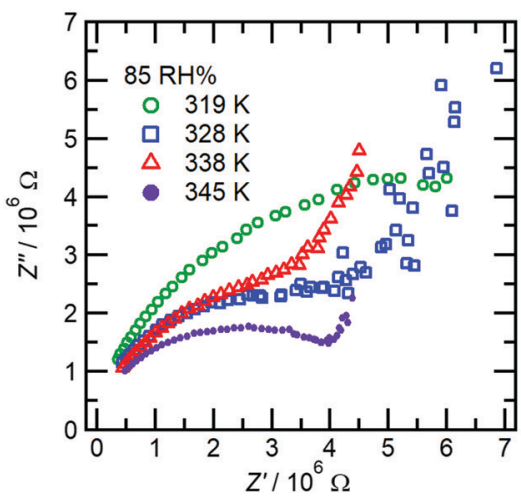

(b)

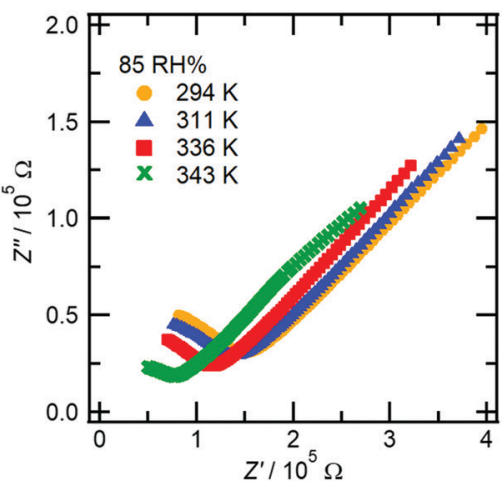

(c)

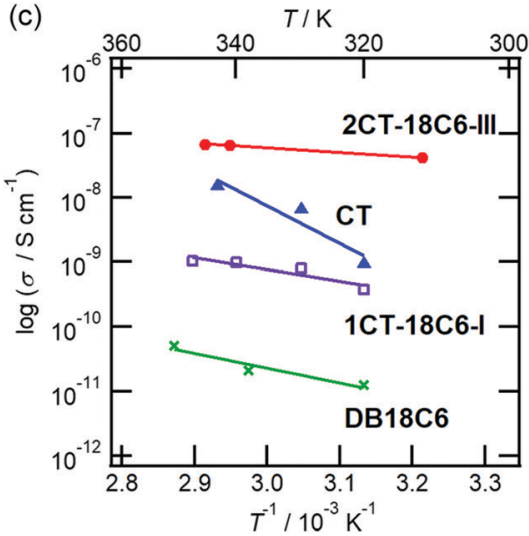

Fig. 6 Proton conductivity for 1CT-18C6-I and 2CT-18C6-III, CT and DB18C6. Nyquist plots for (a) 1CT-18C6-I and (b) 2CT-18C6-III. (c) Arrhenius plot for proton conductivities under $85 \mathrm{RH} \%$.

On the other hand, each Nyquist diagram of DB18C6 was composed of a single semicircle (Fig. S4, ESI $\dagger$ ). Since the pellet sample was used to measure the proton conductivity, the Nyquist plot showed the proton conductions which are contributed from a bulk and an interface. Since bulk conduction is usually observed in the higher frequency range than the interface, ${ }^{11,38}$ the semicircles in the Nyquist diagram of 1CT18C6-I, 2CT-18C6-III, and CT are attributed to bulk conduction. For the calculation of proton conductivity, we applied the equivalent circuit model. The model for the conductivity of DB18C6 is that a resistor and constant phase element (CPE) are arranged in parallel, while the model for that of 1CT-18C6-I, 2CT-18C6-III, and CT is that two parallel pairs of resistors and CPEs were lined up in series.

Regarding the conductivity estimated from the equivalent circuit model, an Arrhenius plot was summarized from the bulk conductivity of 1CT-18C6-I, 2CT-18C6-III, and CT, and the conductivity of DB18C6 (Fig. 6c). From the Arrhenius plot, the proton conductivities at room temperature and $E_{\mathrm{a}}$ of 1CT-18C6-I, 2CT-18C6-III, CT, and DB18C6 were estimated, and summarized in Table 1. The proton conductivity at room temperature was higher in the order of 2CT-18C6-III, 1CT-18C6-I, CT, and DB18C6, and the $E_{\mathrm{a}}$ was also lower in the same order. The proton conductivities of MFM-511 and MFM-512, which are the MOF samples with the carboxy group involved in proton transport, are $4.0 \times 10^{-8}(87 \% \mathrm{RH})$ and $2.5 \times 10^{-7} \mathrm{~S} \mathrm{~cm}^{-1}(77 \% \mathrm{RH})$ at $298 \mathrm{~K}$, respectively. ${ }^{38}$ The value of proton conductivity from 2CT-18C6-III is in the similar order to MFM-511 and MFM-512.

Referring to the proton transport, proton conduction can be classified as the vehicle and the Grotthuss mechanisms, which

Table 1 Proton conductivity at $300 \mathrm{~K}$ and activation energy $\left(E_{\mathrm{a}}\right)$ of 1CT-18C6-I, 2CT-18C6-III, CT, and DB18C6, estimated from the Arrhenius plot

\begin{tabular}{lll}
\hline & $\sigma @ 300 \mathrm{~K} / \mathrm{S} \mathrm{cm}^{-1}$ & $E_{\mathrm{a}} / \mathrm{eV}$ \\
\hline 1CT-18C6-I & $1.95 \times 10^{-10}$ & 0.354 \\
2CT-18C6-III & $3.43 \times 10^{-8}$ & 0.140 \\
CT & $8.69 \times 10^{-11}$ & 1.150 \\
DB18C6 & $3.97 \times 10^{-12}$ & 0.452
\end{tabular}

are divided by $E_{\mathrm{a}}=0.4 \mathrm{eV}$. When $E_{\mathrm{a}}$ is larger and smaller than $0.4 \mathrm{eV}$, proton conductivity is estimated to be by the vehicle and Grotthuss mechanisms, respectively.

Since the $E_{\mathrm{a}}$ values of DB18C6 and CT are higher than $0.4 \mathrm{eV}$, the proton conducting mechanism of DB18C6 and CT is attributed to the vehicle one. On the other hand, 1CT-18C6-I

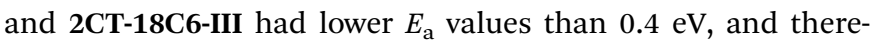
fore, the proton conduction of them is due to the Grotthuss mechanism. Both 1CT-18C6-I and 2CT-18C6-III had continuous hydrogen-bonded networks, resulting in the effective protonconducting pathways and corresponding to the Grotthuss mechanism. Notably, 2CT-18C6-III has two unique linear water pathways. Water molecules in 2CT-18C6-III were not only arranged along the 1D array of the crown ether unit, but also arranged in the parts formed by the hydrogen-bonded networks surrounded by a carboxy group. Therefore, 2CT-18C6-III exhibited the highest proton conductivity of $3.43 \times 10^{-8} \mathrm{~S} \mathrm{~cm}^{-1}$ under $85 \% \mathrm{RH}$ at $300 \mathrm{~K}$.

\section{Conclusions}

In this study, we newly applied 18-crown-6 derivatives with one or two 4,4'-dicarboxy-o-terphenyl (CT) moieties as building block molecules to develop organic crystalline materials with proton conductivity. The 18-crown-6 and carboxy groups capture water molecules via hydrogen bonds and constructed good self-adaptability to water molecule frameworks especially for 2CT-18C6-III with a unique 1D water pathway, which provides available space for proton conduction. Based on this study, it is possible to develop a novel proton conductive flexible framework with good adaptability to guest molecules. In addition, the high chemical stability and superior thermal stability indicate a promising and potential application in PEMFCs.

\section{Conflicts of interest}

There are no conflicts to declare. 


\section{Acknowledgements}

This work was supported by KAKENHI (JP18H01966, JP19H04 557, and JP21H01919) from JSPS and by the Dynamic Alliance for Open Innovation Bridging Human, Environment and Materials from MEXT Japan. X. C. thanks the Chinese Scholarship Council (CSC) for financial support. The authors thank Prof. Y. Sagara (currently at Tokyo Institute of Technology) and N. Tamaoki at RIES, Hokkaido University, for purification of compounds by preparative HPLC. X-Ray diffraction data, including preliminary data sets, were partly collected at BL02B1 and BL40XU in SPring-8 with the approval of JASRI (proposal No. 2019A1161, 2019B1134, and 2020A1117). The authors thank Dr. Kunihisa Sugimoto and Nobuhiro Yasuda at SPring-8 for collection of X-ray diffraction data. The authors also thank the reviewers for their accurate comments.

\section{Notes and references}

1 R. Bashyam and P. Zelenay, Nature, 2006, 443, 63-66.

2 R. Devanathan, Energy Environ. Sci., 2008, 1, 101-119.

3 A. Kraytsberg and Y. Ein-Eli, Energy Fuels, 2014, 28, 7303-7330.

4 R. P. Bisbey and W. R. Dichtel, ACS Cent. Sci., 2017, 3, 533-543.

5 A. I. Cooper, ACS Cent. Sci., 2017, 3, 544-553.

6 H. Zhang and P. K. Shen, Chem. Rev., 2012, 112, 2780-2832.

7 M. Yoon, K. Suh, S. Natarajan and K. Kim, Angew. Chem., Int. Ed., 2013, 52, 2688-2700.

8 S. Bureekaew, S. Horike, M. Higuchi, M. Mizuno, T. Kawamura, D. Tanaka, N. Yanai and S. Kitagawa, Nat. Mater., 2009, 8, 831-836.

9 D. Umeyama, S. Horike, M. Inukai, T. Itakura and S. Kitagawa, J. Am. Chem. Soc., 2012, 134, 12780-12785.

10 J. A. Hurd, R. Vaidhyanathan, V. Thangadurai, C. I. Ratcliffe, I. L. Moudrakovski and G. K. H. Shimizu, Nat. Chem., 2009, 1, 705-710.

11 D.-W. Lim and H. Kitagawa, Chem. Rev., 2020, 120, 8416-8467.

12 K. D. Kreuer, Chem. Mater., 1996, 8, 610-641.

13 W. Weppner, K. D. Kreuer and A. Rabenau, Angew. Chem., Int. Ed. Engl., 1982, 122, 208-209.

14 P. Ramaswamy, N. E. Wong and G. K. H. Shimizu, Chem. Soc. Rev., 2014, 43, 5913-5932.

15 C. J. Pedersen, J. Am. Chem. Soc., 1967, 89, 2495-2496.

16 C. J. Pedersen, J. Am. Chem. Soc., 1967, 89, 7017-7036.

17 G. W. Gokel, W. M. Leevy and M. E. Weber, Chem. Rev., 2004, 104, 2723-2750.

18 M. Bühl and G. Wipff, J. Am. Chem. Soc., 2002, 124, 4473-4480.
19 K. M. Fromm and R. D. Bergougnant, Solid State Sci., 2007, 9, 580-587.

20 Y. F. Han, Y. X. Yuan and H. B. Wang, Molecules, 2017, 22, 266.

21 J. Luo, J. W. Wang, J. H. Zhang, S. Lai and D. C. Zhong, CrystEngComm, 2018, 20, 5884-5898.

22 R. B. Lin, Y. He, P. Li, H. Wang, W. Zhou and B. Chen, Chem. Soc. Rev., 2019, 48, 1362-1389.

23 I. Hisaki, C. Xin, K. Takahashi and T. Nakamura, Angew. Chem., Int. Ed., 2019, 58, 11160-11170.

24 I. Hisaki, J. Inclusion Phenom. Macrocyclic Chem., 2020, 96, 215-231.

25 B. Wang, R.-B. Lin, Z. Zhang, S. Xiang and B. Chen, J. Am. Chem. Soc., 2020, 142, 14399-14416.

26 A. Karmakar, R. Illathvalappil, B. Anothumakkool, A. Sen, P. Samanta, A. V. Desai, S. Kurungot and S. K. Ghosh, Angew. Chem., Int. Ed., 2016, 55, 10667-10671.

27 Y. Qin, T.-I. Gao, W.-P. Xie, Z. Li and G. Li, ACS Appl. Mater. Interfaces, 2019, 11, 31018-31027.

28 Z.-B. Sun, Y.-L. Li, Z.-H. Zhang, Z.-F. Li, B. Xiao and G. Li, New J. Chem., 2019, 43, 1063710644.

29 P. Tholen, C. A. Peeples, R. Schaper, C. Bayraktar, T. S. Erkal, M. M. Ayhan, B. Çoşut, J. Beckmann, A. O. Yazaydin, M. Wark, G. Hanna, Y. Zorlu and G. Yücesan, Nat. Commun., 2020, 11, 3180.

30 Q. Yang, Y. Wang, Y. Shang, J. Du, J. Yin, D. Liu, Z. Kang, R. Wang, D. Sun and J. Jiang, Cryst. Growth Des., 2020, 20, 3456-3465.

31 Y. Wang, J. Yin, D. Liu, C. Gao, Z. Kang, R. Wang, D. Sun and J. Jiang, J. Mater. Chem. A, 2021, 9, 2683-2688.

32 B.-B. Hao, X.-X. Wang, C.-X. Zhang and Q. Wang, Cryst. Growth Des., 2021, 21, 3908-3915.

33 G. R. Desiraju, Angew. Chem., Int. Ed. Engl., 1995, 34, 2311-2327.

34 K. Kobayashi, T. Shirasaka, E. Horn and N. Furukawa, Tetrahedron Lett., 2000, 41, 89-93.

35 I. Hisaki, S. Nakagawa, N. Ikenaka, Y. Imamura, M. Katouda, M. Tashiro, H. Tsuchida, T. Ogoshi, H. Sato, N. Tohnai and M. Miyata, J. Am. Chem. Soc., 2016, 138, 6617-6628.

36 I. Hisaki, Y. Suzuki, E. Gomez, Q. Ji, N. Tohnai, T. Nakamura and A. Douhal, J. Am. Chem. Soc., 2019, 141, 2111-2121.

37 D.-W. Lim, S. A. Chyun and M. P. Suh, Angew. Chem., Int. Ed., 2014, 53, 7819-7822.

38 P. Rought, C. Marsh, S. Pili, I. P. Silverwood, V. G. Sakai, M. Li, M. S. Brown, S. P. Argent, I. Vitorica-Yrezabal, G. Whitehead, M. R. Warren, S. Yang and M. Schröder, Chem. Sci., 2019, 10, 1492-1499. 